\title{
Adjustable Window for Amplitude Estimation Considering the Time-Varying Frequency of Power Systems Signals.
}

\author{
Thiago R.F. Mendonça, Milena F. Pinto and Carlos A. Duque. \\ Federal University of Juiz de Fora - UFJF \\ Juiz de Fora, MG - Brazil \\ thiago.mendonca@engenharia.ufjf.br
}

\begin{abstract}
The development of signal processing techniques that allows the estimation of parameters from the power system are crucial to keep the grid within a safe margin of operation. Some methods require a specific sampling rate in order to avoid asynchronous sampling, which may result in errors in estimation algorithms. Due to unbalances between demand and supply, the power signal is time-varying in nature, hindering the selection of an optimal fixed window length. In this work is proposed a technique in which the window length adapts to the actual value of estimated frequency, achieving better performance. The filter utilized to test the method was a moving average filter, but it can be expanded for other algorithms. Results have shown good performance when changing the filter coefficients according to frequency estimation.
\end{abstract}

Keywords - Adjustable Filter, Frequency Oscillation, Modified Moving Average Filter, Parameter Estimation, Variable Window Length.

\section{INTRODUCTION}

Power quality issues brought by the widespread use of nonlinear electronic equipment are of great concern among industries, consumers and utilities. In this sense, the estimation of amplitude, spectrum content, fundamental frequency and phase from the electrical power system's signals are of extreme importance to keep relevant parameters within regulated limits. Additionally, the recent modernization of the power grid toward the smart grid concepts, demands improved monitoring capability, which relies in novel methods of signal processing techniques [1], justifying this area of research. The degree of accuracy for measuring and detecting relevant events related to the power quality are discussed in some standards and amongst them is cited the IEC 61000 [2], which defines assessment for conformity and identify vulnerability from the power grid. In order to extract these useful parameters from the power system signal to assure a safe and reliable operation, one may utilize several well-known signal processing algorithms [3].

However, the great majority of techniques require a specific sampling rate in order to avoid asynchronous sampling, achieving in this way better results [4]. Due to imbalance between demand and supply, the power signal is time-varying in nature, hindering the selection of an optimal fixed window length. Even though the permissible tolerances of known standards are around $\pm 0.5 \mathrm{~Hz}$, this small deviation is sufficient to cause considerable errors in estimation approaches.
It is possible to overcome the improper sampling issue by varying the sampling period or the window length of the observable data, as can be seen in [5] and [6], respectively. However these methodologies are still under developed, presenting some implementation difficulties such as the practical problems for storing and analyzing the variable sampled signal and fractional window lengths.

In this way, it is proposed in this paper an improvement of the variable window length algorithm that adjusts itself by considering the estimated frequency at each iteration and that comprises an interpolator structure, overcoming difficulties imposed by fractional lengths. To validate the adjustable vector length algorithm and evaluate how a filter with variable coefficients behaves with several transient conditions, a Moving Average Filter (MAF) is utilized for amplitude estimation and fundamental component reconstruction. It is noteworthy that this algorithm is heavily dependent on reliable frequency estimators, since it interferes directly in the calculation of new window size.

This paper is organized as follows. The second section discusses the recursive moving average filter with fixed coefficient. Section III presents the methodology for adapting the filter's coefficients for optimal estimation when the fundamental frequency varies. In section IV is shown the results considering both algorithms, fixed and adjustable coefficients, proving that the proposed algorithm achieved better results.

\section{Moving Average FiLter Design With FiXed Window}

This is one of the simplest digital low pass filters, which computes the average of "L" samples, updating its buffer in every iteration. The equation that computes the average of $\mathrm{L}$ past samples is presented in (1).

$Y[n]=\frac{X[n]+X[n-1]+X[n-2]+. .+X[n-L+1]}{L}$

By performing some simple mathematical manipulations, it is possible to rewrite (1) in a recursive form as presented in (2). This process is described in details in reference [7]. For the z- 
domain representation, it is sufficient to consider each delay in the time-domain as $z-1$, resulting in (3).

$$
\begin{aligned}
& Y[n]=Y[n-1]+\frac{X[n]-X[n-L]}{L} \\
& Y(z)=Y(z) \cdot z^{-1}+\frac{X(z)-X(z) \cdot z^{-L}}{L}
\end{aligned}
$$

The transfer function can be calculated considering the ratio between the output $\mathrm{Y}[\mathrm{z}]$ and the input $\mathrm{X}[\mathrm{z}]$. From (3), it is possible to obtain the transfer function of the moving average filter, which is presented in (4). Note that it exists only one pole different than zero, located at $Z=1$, and from the Moivre theorem, Z-L results in $\mathrm{L}$ complex roots equally spaced. The pole in $Z=1$ forces the pass band to be in the low frequency region.

$$
\frac{Y(Z)}{X(Z)}=H_{M A F}(Z)=\frac{1}{L} \cdot \frac{1-Z^{-L}}{1-Z^{-1}}
$$

The number of samples to perform the average directly affects the cutoff frequency of the overall system. It has particular application within power systems due to the possibility to eliminate harmonics when the sampling frequency and the value of "L" are chosen correctly. Considering for example a situation where a signal is sampled at 16 samples per cycle and $\mathrm{L}$ is chosen as 16 , then the magnitude response as well as the poles and zeros localization in the complex circle is as presented in Fig. 1 and Fig. 2, respectively. Note that the pass band is in the DC component of the signal and has a cutoff frequency for every integer multiple of $60 \mathrm{~Hz}$.

Once the filter's coefficients are defined, it will remain fixed independently of the actual value of the signal frequency and therefore, will no longer eliminate unwanted harmonics with good performance, only attenuating it. Another issue to address when using this kind of filter for amplitude estimation of power systems signals is that, since it is a low pass filter, it is necessary to modulate the original signal in order to shift the fundamental component to its passband.

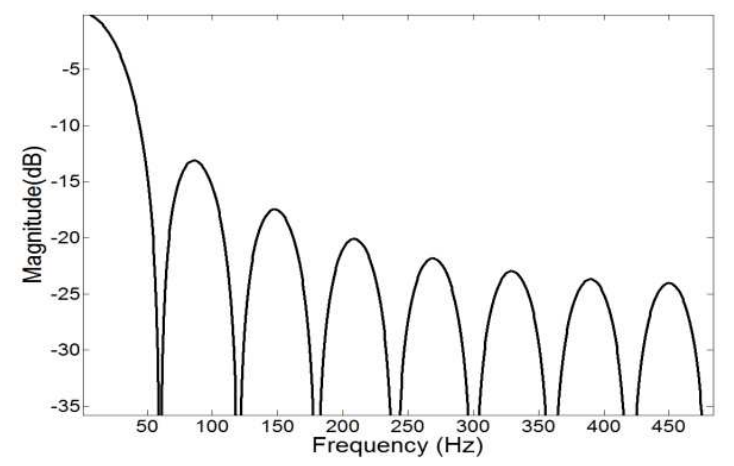

Fig. 1. Magnitude response of a MAF with window length equal to the number of samples per cycle.

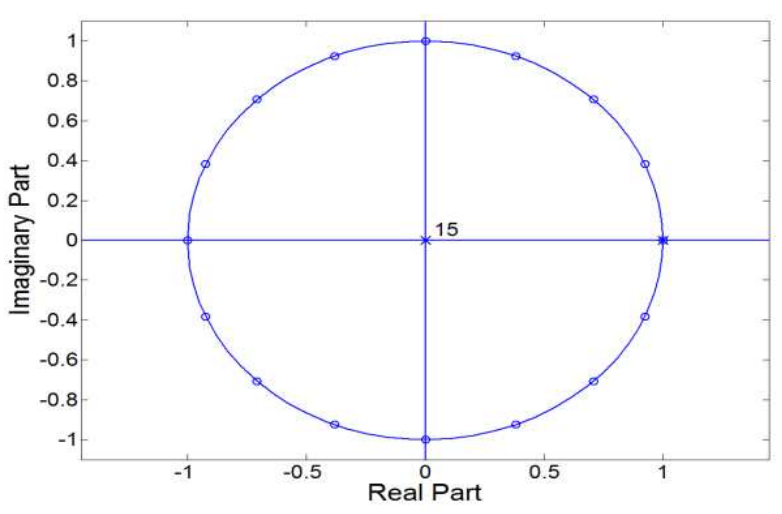

Fig. 2. Poles and zeros representation in the complex circle.

\section{Proposed Algorithm FOR AdJUSTING THE WINDOW LENGTH}

A common error while applying signal processing techniques to analyze power system signals is the selection of the observable data. It is known that in order to obtain better performance in several algorithms, the number of samples utilized must be inside an integer number of cycles. This is not an easy task due to the time-varying nature of the signal.

It is discussed in references [8]-[11] some methods for reducing the error when asynchronous sampling occurs. Another form to increase the estimation process performance is to avoid this condition, which can be achieved by either working with a variable sampling rate or with a variable window length, both updated by the current frequency estimation. The difficulties imposed by the adjustable sampling rate are due to the complexity for hardware implementation and data recording for offline analysis, while the adjustable window algorithm may result in a fractional length and therefore, still output estimations with errors. This work presents a form to enhance the variable window length method by adjusting the size of the observable data, inserting an interpolator structure to the moving average design.

\section{A. Adjustable Window Method}

In this section, an implementation of a flexible window length to be used in parameters estimation algorithms is presented. To validate the methodology, this technique is applied in a moving average filter (MAF) in order to estimate amplitude as well as to reconstruct the fundamental component from a typical frequency varying signal. This method consists in changing the size of the observable data based on the frequency estimation using the relation presented in (5), where $\mathrm{f}$ is the fundamental frequency of the correspondent signal and the parameter Ts is the sampling interval.

$$
\mathrm{L}=\frac{1}{\mathrm{~T}_{\mathrm{S}} \cdot \mathrm{f}}
$$

In this way, a new window length is computed for each frequency output. In order to obtain the amplitude estimation and the desired component reconstruction with a low pass filter, it is first required to modulate the signal in order to shift it to the passband. Since the power system signal is timevarying, the frequency estimator updates the modulator 
accordingly. The MAF algorithm is used with the modulated signal and as result, the quadrature and in phase components are computed. This procedure is depicted in Fig. 3.

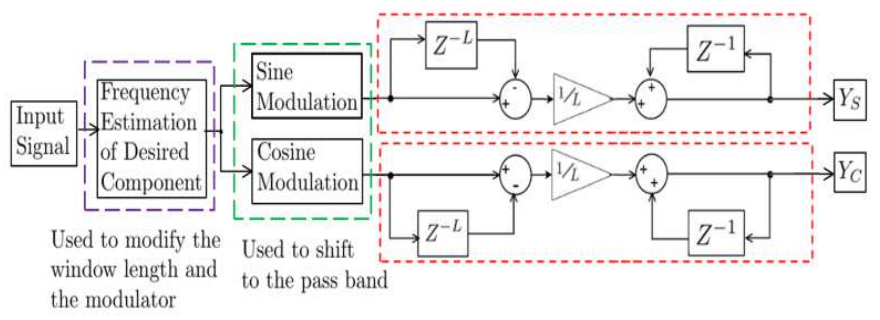

Fig. 3. Diagram for computing the in phase and quadrature components for amplitude estimation and component reconstruction.

The first traced block concerns the frequency estimator algorithm (e.g. phasor rotating, zero crossing, phase locked loop, among others), which will be used to modify the MAF coefficients and also to change the modulator. Since the phase angle of the analyzed signal may vary, it is necessary to perform the modulation with a sine and with a cosine function to obtain the in phase and quadrature components. The third traced block is the recursive implementation of the moving average filter presented in (4), where L is the computed window length.

The amplitude estimation of the sampled signal can be calculated by the norm of YC and YS. The desired component reconstruction is calculated by adding the modulated versions of the output of the MAF structure of Fig. 3. It is important to note that the modulator must be set with the same frequency of the first modulator. The amplitude estimation and signal reconstruction is presented in Fig. 4

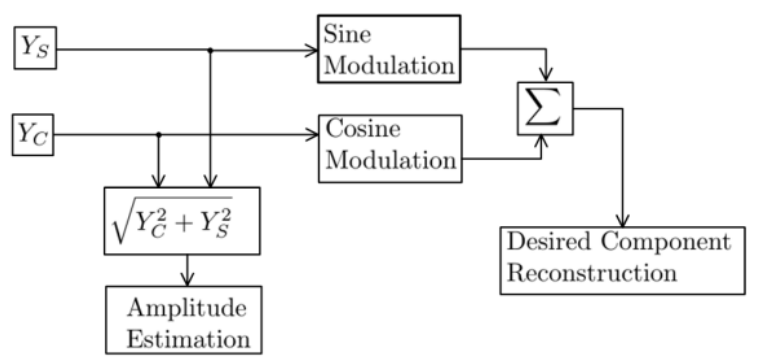

Fig. 4. Diagram for estimating the amplitude of the signal and reconstruct the desired component.

To achieve a variable window length, this method relies on a fixed and oversized buffer in order to comport the information of past samples to be used in the MAF algorithm. It must be oversized so that when frequency decreases, according to (5) the observable vector will increase and must still fit in the buffer. In Fig. 5 is presented an example of the variable window length with a fixed and oversized buffer. The nominal value of "L" is 6 . After an increase in frequency estimation, it is known that the number of samples within a complete cycle decreases, so now "L" is 5 and therefore, only the first five elements stored in the buffer will be used in the algorithm. With a decrement in the system frequency, the new "L" is higher. Therefore, in this fictional example, the window length needs to be 7 , so the first seven elements of the buffer will be used in the algorithm. It is noteworthy that the buffer updates in real time.

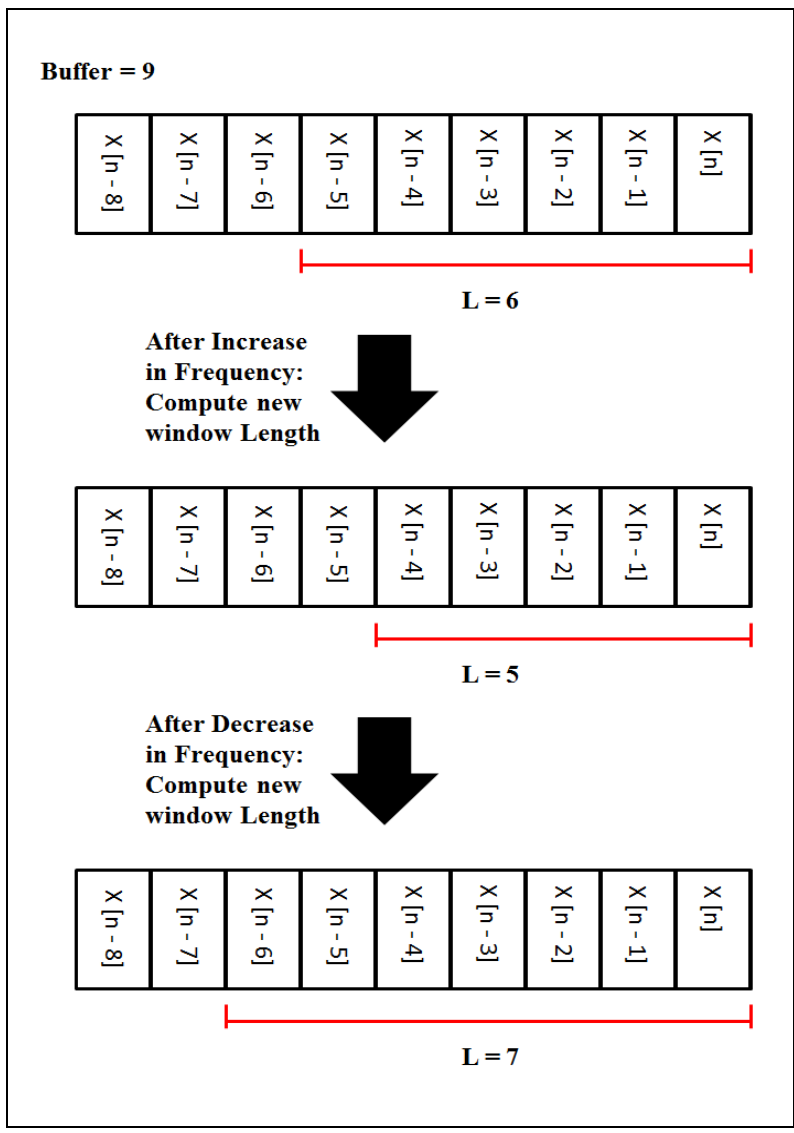

Fig. 5. Fixed and oversized buffer for implementing the variable window length algorithm.

A common problem in applying the variable window technique is when the result is a fractional length, which is usually just rounded to the closest value. This procedure still presents large values of error in oscillatory frequency cases, such as in the power system. This work proposes an interpolating structure to achieve better performance with minor alteration in the implementation form presented in Fig. 3.

\section{B. Interpolation of the Fractional Window Length in a Moving Average Filter}

The frequency in a power system signal is allowed to oscillate around $0.5 \mathrm{~Hz}$ in normal conditions [2]. Depending on the sampling rate, this small deviation may not be enough to cause an integer change in the window length to fit a complete cycle. So it is of great relevance to interpolate whenever the length results in a fractional value. For high sampling rate, a simple linear interpolation can be utilized, achieving low errors. In Fig. 6 is presented an illustration of 
a linear interpolation case where "L" is the rounded length, "Lf" is the fractional window length and " $n$ " is the current sample. In this way, the estimation of the signal value in the fractional sample is performed based on the delayed by the integer "L" and "L-1" samples, which are both known and stored in the buffer. It is worth to notice that the linear interpolation also presents some error due to the linear approximation of the original signal in the interval of two consecutive samples.

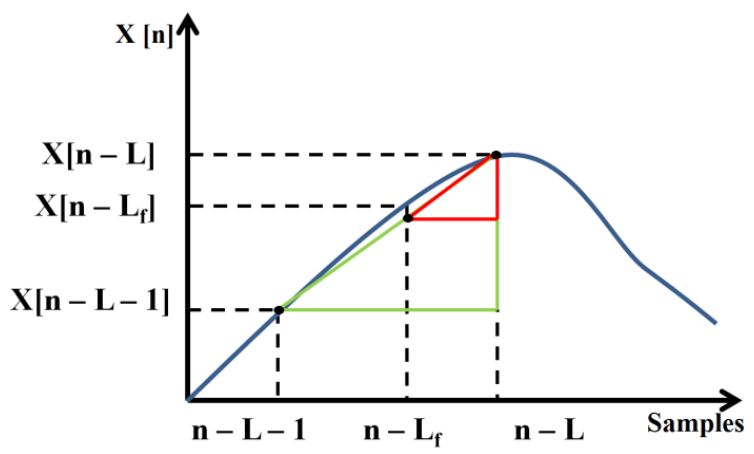

Fig. 6. Linear interpolation of the fractional window length.

By analyzing the red and green triangles, it is possible to write an expression to calculate $\mathrm{x}[\mathrm{n}-\mathrm{Lf}]$ as a function of its delayed by $\mathrm{L}$ and L-1 values. The equations presented in (6) and (7) describe this process.

$$
\frac{X[n-L]-X[n-L-1]}{1}=\frac{X[n-L]-X\left[n-L_{f}\right]}{L_{f}-L}
$$

Separating the term X[n - Lf] and writing the constant Lf-L as Lfrac it is possible to obtain (7).

$$
X\left[n-L_{f}\right]=X[n-L]-L_{\text {frac }} \cdot(X[n-L]-X[N-L-1])
$$

In (7) is stated how to use the linear interpolated value of the signal delayed by fractional "Lf" samples using data from the buffer. This must be incorporated to the moving average filter design presented in (2) changing the term of $\mathrm{X}[\mathrm{n}-\mathrm{L}]$ to $\mathrm{X}[\mathrm{N}-\mathrm{Lf}]$. The recursive form in a diagram representation is shown in Fig. 7, where "Lfrac" is the constant equal to the difference between the fractional length and the rounded version and "Lf".

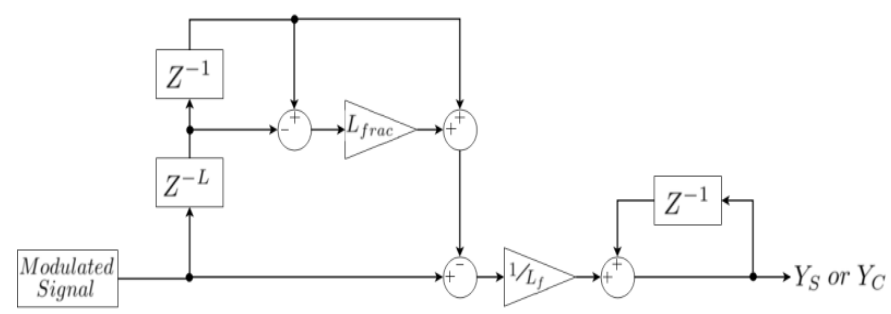

Fig. 7. Diagram for implementing the linear interpolator for variable window length applied to a moving average filter.

To obtain the moving average filter with variable window length, considering the interpolation process for optimal amplitude estimation as well as fundamental component reconstruction, the traced red block depicted in Fig. 3 must be substituted by the diagram of Fig. 7 .

With the adjustable window implemented in a fixed and oversized buffer, accounting the algorithm for modulating and interpolating fractional windows, it is possible to estimate the amplitude of a signal with oscillating frequency with better performance. The following section presents the results obtained as well as comparisons with the conventional method under some specific cases with variable frequency and amplitude of a sinusoidal signal.

\section{RESULTS}

It is analyzed two types of signal in order to compare performances between the proposed method and the conventional one for estimating amplitude. In both cases, there is a step change in frequency from 60 to $61 \mathrm{~Hz}$. However, in the second there is also a decrease in voltage for a brief period of time to evaluate the amplitude tracking capability.

The sampling rate chosen for the experiments is of 128 samples per cycle of a $60 \mathrm{~Hz}$ fundamental frequency, i.e. 7680 samples per second. The conventional MAF is designed with a fixed window length containing 128 samples. In the proposed design is utilized a variable number of samples proportional to the signal estimated frequency according to (5). The frequency estimator algorithm used is the simple zero crossing approach, which detects consecutive passage through zero and compute the time between them to determine the system's frequency. A low pass filter is added in the output of the frequency estimator in order to obtain a smooth curve. The estimator performance compared to the real value can be seen in Fig. 8. It is noteworthy that an abrupt change in the systems frequency is not real. However, a step change is interesting for analyzing steady state and transient behavior of the proposed methodology.

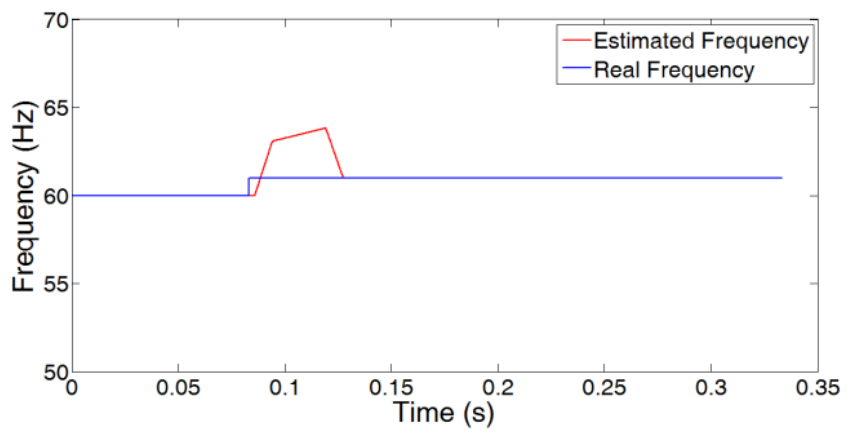

Fig. 8. Frequency estimation in the case with step change in frequency, using zero crossing technique.

The amplitude estimation of the sinusoidal signal with step change in frequency using the conventional and the proposed method can be visualized in Fig. 9 and Fig. 10, respectively. The abrupt transition in frequency causes an oscillation in the filter output and, therefore, a reset of the 
buffer is required in both cases. Otherwise, some small error may be present in the steady state condition.

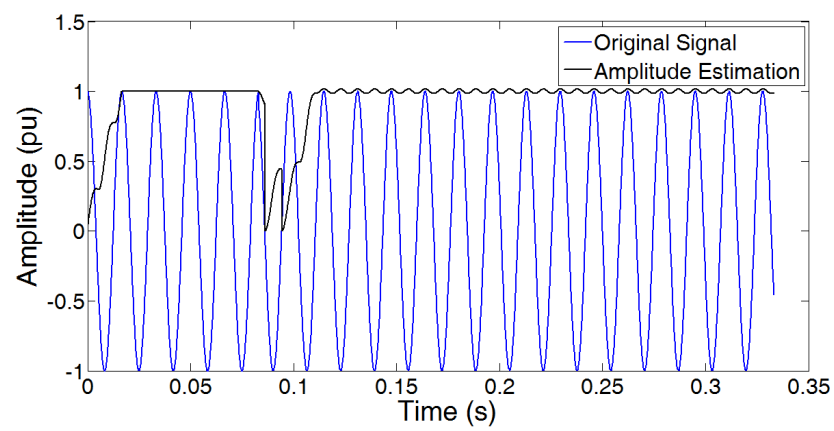

Fig. 9. Amplitude estimation in the case with step change in frequency using the conventional algorithm.

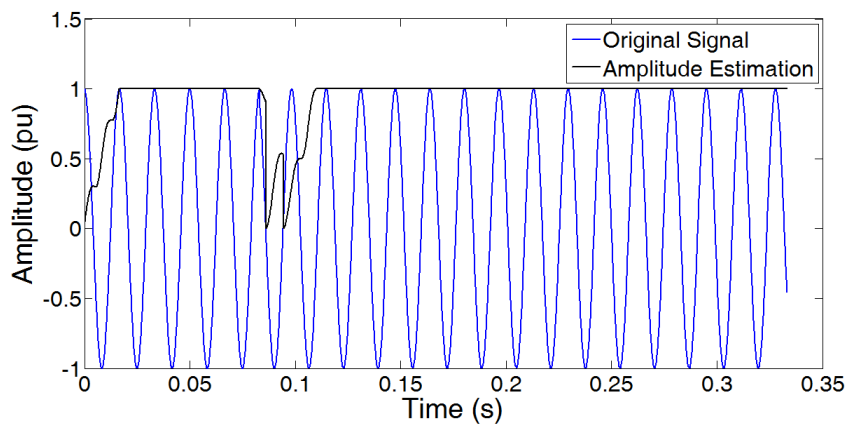

Fig. 10. Amplitude estimation in the case with step change in frequency using the proposed algorithm.

Note the oscillation present in the amplitude estimation using the conventional method after the frequency changes from 60 to $61 \mathrm{~Hz}$. This is caused by the filter's coefficients that were selected based on a $60 \mathrm{~Hz}$ fundamental frequency. With the proposed method, this effect is almost negligible, reaching errors around $0.004 \%$ compared to errors around $1.5 \%$ achieved with the conventional method. The comparison of errors obtained with both algorithms considering the same test signal is presented in Fig. 11. The error obtained with the proposed method is shown in more detail in Fig. 12, with a proper axis dimension.

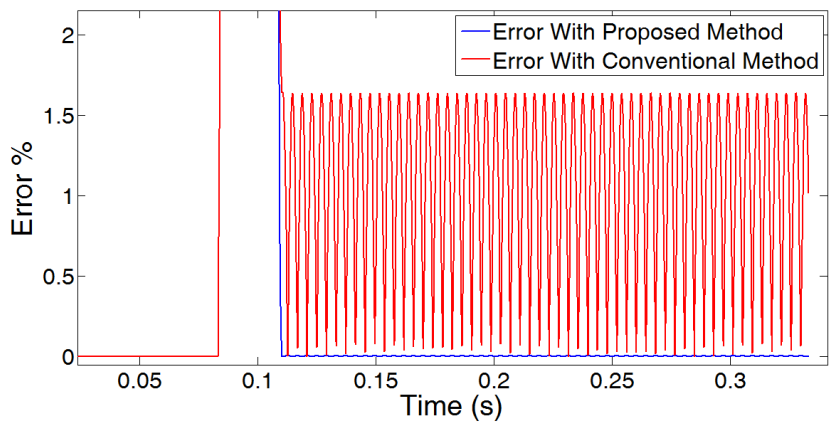

Fig. 11. Comparison of the error obtained with the proposed method and the error obtained with the conventional method for the first case.

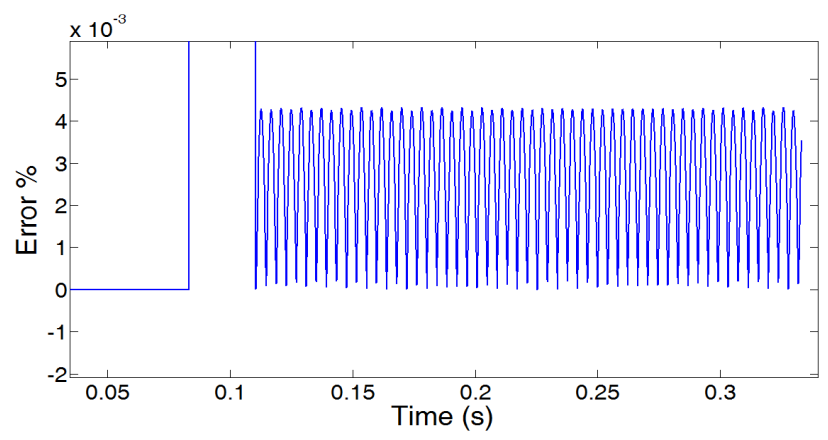

Fig. 12. Error of amplitude estimation in the case with step change in frequency using the proposed method.

Beside the step change in frequency, the second case also contains a momentary decrease in the signal amplitude, characterized by voltage dips in power systems applications. This test is useful to evaluate the capability to track the signal's amplitude. The test signal as well as the amplitude estimation is depicted in Fig. 13.

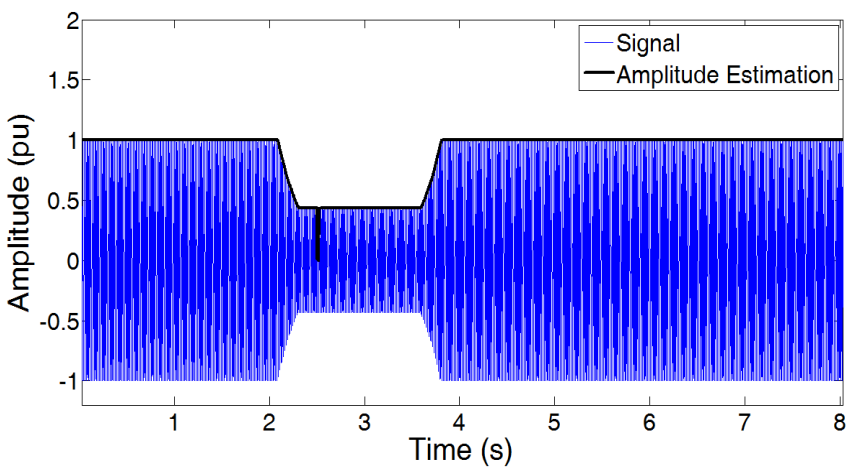

Fig. 13. Amplitude estimation of a signal with step change in frequency as well as signal dip.

It is expected some error during the transition in amplitude, since the algorithm presents some delay. However, during the steady state condition, it is desired to have a more precise estimation. The error curve for the amplitude estimation using the conventional method is depicted in Fig. 14. Note that before 2 seconds, the error is null since the fundamental frequency is the same as the one used for defining the filter's coefficients. During the exponential decrease in amplitude, the error increases due to the delay. The abrupt change in frequency causes the peak in error. It can be noted that after the frequency change, the conventional method always carries an error around $1.5 \%$ and increases when the amplitude starts varying.

The error obtained in the proposed method, for this same case is presented in Fig. 14. The error obtained when the frequency is $60 \mathrm{~Hz}$ is fairly close to the error obtained when the system's frequency changed to $61 \mathrm{~Hz}$, validating the capability to adjust the filter's coefficients to the actual frequency estimation provided from the zero crossing algorithm. The same delay issue is also present, resulting in high errors during the varying amplitude. However, it is 
possible to see that while the voltage is increasing with frequency of $61 \mathrm{~Hz}$, the error obtained from the proposed method is lower than the one obtained by the conventional one. After the amplitude is constant and frequency is different from nominal, the adjusted coefficients results in errors near to zero.

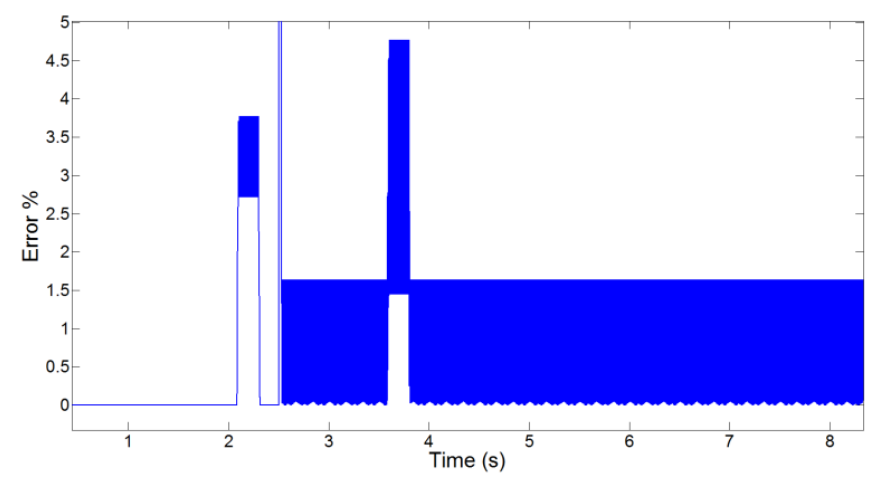

Fig. 14. Error of amplitude estimation in the case with step change in frequency and sag event using the conventional method.

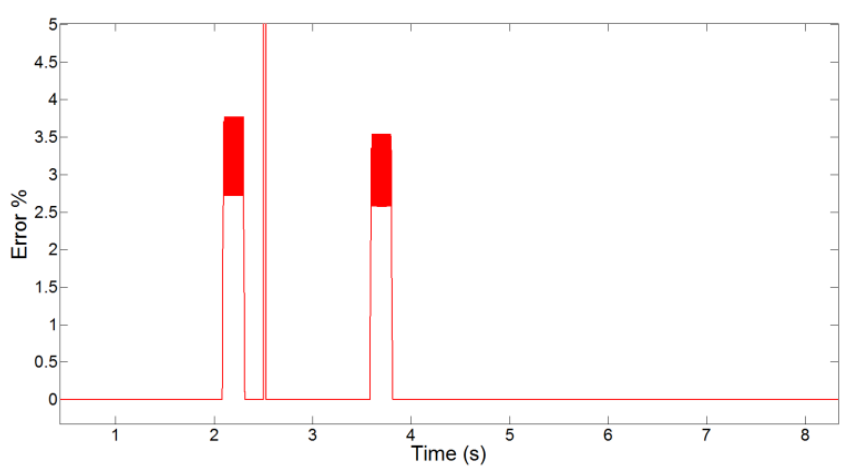

Fig. 15. Error of amplitude estimation in the case with step change in frequency and sag event using the proposed method.

It is possible to note from comparing both figures that the time interval with high value of error due to the delay for tracking amplitude is the same for both algorithms. However, the error using the proposed method is much lower than the obtained by the conventional design.

\section{V.CONCLUSION}

In order to present the proposed method for adjusting the window length according to the estimated frequency of the signal, a brief review of amplitude estimation with moving average filter was presented as well as the algorithm for implementing a variable window length. The proposed work has improved this technique by interpolating whenever the length results in a fractional length.

A test has been performed using a moving average filter applied in two test signals, both with a step change in frequency from $60 \mathrm{~Hz}$ to $61 \mathrm{~Hz}$. However, in one case the amplitude kept constant, while the other contained a sag event.
Results have shown good performance for amplitude estimation when changing the filter coefficients as the frequency varies, without representing high computational effort. It is noteworthy that the tracking capability is still not perfect, reaching errors around 3\%. However, others well-known algorithms also have the same issue.

For future works, it is intended to analyze performance with other interpolating functions, evaluating computational complexity and performance. Additionally, it is desired to implement this technique in other parameter estimation algorithms to evaluate how it could behave.

\section{ACKNOWLEDGMENT}

The authors would like to thank the Federal University of Juiz de Fora, CAPES, FAPEMIG, CNPq, INERGE and the Brazilian Government for all financial, technical and scientific support on this work.

\section{REFERENCES}

[1] C.A.G, Marques; M.V, Ribeiro; E.A.B, da Silva. "Enhanced demodulation-based technique for estimating the parameters of fundamental component in power systems" Generation, Transmission \& Distribution, IET, vol. 5, 2011

[2] International Electrotechinical Commission IEC 61000-4-30 "testing and measurement techniques - Power quality measurements methods" 2003.

[3] P. Stoica, H. Li and J. Li, , "Amplitude Estimation of Sinusoidal Signals : Survey, New Results, and an Application," 338 IEEE Trans. Signal Process., vol. 48, no. 2, pp. 338-352, 2000.

[4] A. Testa, D. Gallo and R. Langella, "On the Processing of Harmonics and Interharmonics: Using Hanning Window in Standard Framework," IEEE Transactions On Power Delivery, vol. 19, no. 1, 2004.

[5] G. Benmouyal. "An Adaptive Sampling-Interval Generator for Digital Relaying,” IEEE Power Eng. Rev., vol. 9, no. 7, pp. 45-46, 1989.

[6] D. Hart, N. Damir, Y. Hu, B. Smith, and M. Egolf, "A New Frequency Tracking and Phasor Estimation Algorithm for Generator Protection," IEEE Trans. Power Deliv. Vol. 12, No. 3, July 1997, vol. 12, no. 3, pp. 1064-1073, 1997.

[7] S. K. Mitra and Y. Kuo, “ Digital signal processing: a computer-based approach,"vol. 2, New York: McGraw-Hill, 2006.

[8] Z. Liu, J. Himmel and K. W. Bonfig, "Improved processing of harmonics and interharmonics by time-domain averaging," Power Delivery, IEEE Transactions on, vol.20, no.4, pp.2370-2380, Oct. 2005.

[9] G. W. Chang and Cheng-I Chen, "Measurement techniques for stationary and time-varying harmonics," Power and Energy Society General Meeting, 2010 IEEE, vol., no., pp.1-5, 25-29 July 2010.

[10] J. Valenzuela and J. Pontt, "Real-time interharmonics detection and measurement based on FFT algorithm," Applied Electronics, pp.259264, 9-10 Sept. 2009.

[11] D. Gallo, R. Langella and A. Testa, "On the processing of harmonics and interharmonics in electrical power systems," Power Engineering Society Winter Meeting, IEEE, vol.3, pp.1581-1586 vol.3, 23-27 Jan 2000 . 